\title{
Pluto in Glory: Discovery of its Huge Opposition Surge
}

B. J. Buratti (1), M. D. Hicks (1), E. Kramer (1), J. Bauer (2), D. R. Ciardi (3), M. B. Lund (3), K. J. Lawrence (1)

(1) Jet Propulsion Laboratory, California Institute of Technology; (2) University of Maryland;

(3) Infrared Processing Analysis Center, California Institute of Technology

Copyright 2021. California Institute of Technology

\section{Key Points:}

Pluto reached an historically small solar phase angle that will not be repeated for 161 years and that complements New Horizons observations.

Observations with the adaptive optics system at Palomar Observatory during this time successfully captured both hemispheres of Pluto.

Pluto exhibits an unusually large opposition surge that may be due to active geology and an unusual surface texture.

This article has been accepted for publication and undergone full peer review but has not been through the copyediting, typesetting, pagination and proofreading process, which may lead to differences between this version and the Version of Record. Please cite this article as doi: 10.1029/2021GL092562.

This article is protected by copyright. All rights reserved. 


\begin{abstract}
Near-infrared observations of the Pluto Charon system were captured with the Palomar High Angular Resolution Observer (PHARO) adaptive optics system on the 200-inch Hale telescope during the historically small solar phase angles in 2018-2019. Both objects exhibit large opposition surges of $\sim 30-35 \%$ in the last half degree of solar phase angle, which is among the largest observed for icy moons and other Kuiper Belt Objects. In addition, Pluto's surge is exceptionally steep. Pluto's unusual phase curve may be due to an unusual surface texture caused by seasonal volatile transport and active geologic processes. These observations enable accurate determination of Pluto's geometric albedo in the JHK filter system, which we find to be $0.86 \pm 0.04,0.59 \pm 0.05$, and $0.39 \pm 0.04$ for Pluto, respectively, and $0.68 \pm 0.06$ for Charon in the J-filter.
\end{abstract}

\title{
Plain Language Summary
}

The New Horizons spacecraft revealed Pluto to be a geologically active, complex world. It never observed small solar phase angles - when the disk of Pluto is fully illuminated. In 2018 and 2019, Pluto reached an historically small solar phase angle that will not be repeated for 161 years. Observations at Palomar Observatory show that both hemispheres of Pluto, as well as its main moon Charon, exhibit a surge of over $30 \%$ in brightness at opposition, suggesting an unusual surface texture due to activity and volatile transport.

\section{Introduction}

The July 2015 encounter of the New Horizons spacecraft with Pluto brought a large Kuiper Belt Object into sharp focus for the first time (Stern et al., 2015; Moore et al., 2016; Grundy et al., 2016a; Gladstone et al., 2016; Buratti et al., 2017). The "dwarf planet" is a dynamic body with the first-ever observed active glaciers outside the Earth, possible clouds, snow, haze, seasonal volatile transport, and at least two examples of the dark, elusive material that is ubiquitous in the outer Solar System and that may be tied to the origin of life on Earth. But as spectacular as this flyby was, it represented an instant in time, leaving out the long temporal baseline that is required to capture, understand, and model the seasonal events and the types of geologic processes that were observed on Pluto - and that happen on 100-year time scales, at least. As with most other flyby encounters, entire viewing geometries and spectral domains were lacking, as the puzzling X-ray observations of Pluto by Lisse et al. (2017) showed.

One key observation that was not observed by New Horizons and that can only be observed in 2018-2022 - the next opportunity does not occur for another 161 years - is the Pluto-Charon system at true opposition: the lowest possible solar phase angles that occur when Pluto is at a nodal crossing of the orbital planes of Earth and Pluto and the Earth is also at the longitude of Pluto's ascending or descending node.

It is well known that the Moon exhibits a large non-linear increase in brightness as it reaches this full phase. The canonical explanation of this "opposition surge" is that it is caused by the rapid disappearance of mutual shadowing among particles comprising the optical portion of the regolith (Irvine, 1966; Hapke, 1986; 2008). In the 1980's and 1990's several icy moons of the outer planets were observed to exhibit an even narrower large surge in brightness at solar phase angles less than $1^{\circ}$ (Domingue et al., 1991; Brown et al., 1983; Buratti et al.,1992). Clementine observations of the Moon revealed a similar surge, suggesting that it is characteristic of rocky bodies as well as icy

This article is protected by copyright. All rights reserved. 
ones (Buratti et al., 1996). This sharp peak was explained in terms of coherent backscatter, in which multiply scattered photons following the same but reversed paths interfere constructively in the backscatter direction to cause an increase in brightness (Hapke, 1990, 2009; Mishchenko, 1992, 2009; Ozrin, 1992). Neptune's moon Triton, which is similar to Pluto in size with a visible geometric albedo of 0.77 and is most likely a captured Kuiper Belt Object, exhibits a visual opposition surge of 0.20 magnitudes in the last 0.5 degree of its solar phase curve, with a minimum observed phase angle of $0.02^{\circ}$ (Buratti et al., 2011).

This paper reports on successful observations during the historically small solar phase angles of both hemispheres of Pluto and Charon with the near-infrared camera Palomar High Angular Resolution Observer (PHARO; Hayward et al. 2001) behind the P3K adaptive optics (AO; Dekany et al., 2013) system on the 200-inch Hale telescope. This AO system covers the J, H, K filters corresponding to $1.25,1.65$, and $2.22 \mu \mathrm{m}$. Both Pluto and Charon exhibit opposition surges of $\sim 30-35 \%$ over the last half-degree of solar phase angle, with a minimum phase angle of $0.0084^{\circ}$ observed on July 12, 2018.

\section{Observations and Data Analysis}

Data were gathered over 5 nights spanning the lowest solar phase angles in 2018 and 2019, corresponding to the two hemispheres of Pluto: the bright "heart" of Pluto - Tombaugh Regio in 2019, and the opposite hemisphere that was poorly imaged by New Horizons, in 2018. We used the Hale 200-inch Telescope's PHARO adaptive optics system on the nights listed in Table1 in the imaging mode and resolution set to 40 milliarcseconds, giving a field of view of approximately 40 arcseconds. We were assigned four half nights in 2018 and obtained data on all four; on July 29 we were not able to resolve the two bodies because of poor seeing. In 2019 we received seven half nights; five of the nights were plagued by weather and poor seeing and one equipment issue, but good observations were obtained on July 12 and 13, when the system was at its historic low solar phase angle for the higher albedo hemisphere of Pluto covering Tombaugh Regio. Typical integration times were 80 seconds for all filters. We obtained daytime dark frames at each integration time utilized for observations. Flatfields in each of the J, H, and K filters were obtained with an illuminated dome. Table 1 summarizes the successful observations along with geometric parameters and the results. (The geometric parameters are $\mathrm{r}=$ heliocentric range, $\Delta=$ observer range, and $\alpha=$ average solar phase angle for each night.) On the night of the minimum solar phase angle $\left(0.0084^{\circ}-0.011^{\circ}\right)$ we nearly reached the theoretical limit of the smallest effective solar phase angle observable, which is set by the angular radius of the Sun at the target to be $0.0079^{\circ}$, which was visible at Palomar only during daylight hours.

Table 1 - Summary of observations

\begin{tabular}{|l|l|l|l|l|l|l|l|l|l|}
\hline Date & $r$ & $\Delta$ & $\alpha$ & $\begin{array}{l}\text { Longitude }^{1} \\
\text { Latitude }\end{array}$ & $\#$ Images & $\begin{array}{l}\text { Pluto J } \\
\text { brightness }\end{array}$ & $\begin{array}{l}\text { Pluto H } \\
\text { brightness }\end{array}$ & $\begin{array}{l}\text { Pluto K } \\
\text { brightness }\end{array}$ & $\begin{array}{l}\text { Charon J } \\
\text { brightness }\end{array}$ \\
\hline$(\mathrm{UT})$ & $(\mathrm{AU})$ & $(\mathrm{AU})$ & $\left(^{\circ}\right)$ & $\left(^{\circ}\right)$ & $(\mathrm{J} / \mathrm{H} / \mathrm{K})$ & $\mathrm{mag}^{2,3}$ & $\mathrm{mag}^{2,3}$ & $\mathrm{mag}^{2,3}$ & $\mathrm{mag}^{2,3}$ \\
\hline $\begin{array}{l}20180712 \\
7: 02-9: 23\end{array}$ & 33.60 & 32.58 & 0.010 & $\begin{array}{l}39 \\
54.9\end{array}$ & $18 / 12 / 7$ & $\begin{array}{l}13.66 \pm 0.05 \\
(13.65)\end{array}$ & $\begin{array}{l}13.77 \pm 0.09 \\
(13.78)\end{array}$ & $\begin{array}{l}14.1 \pm 0.1 \\
(14.12)\end{array}$ & $15.35 \pm 0.04$ \\
& & & & & & & \\
& & & & & & & \\
\hline $\begin{array}{l}20180713 \\
6: 18-9: 20\end{array}$ & 33.60 & 32.58 & 0.021 & $\begin{array}{l}342 \\
54.9\end{array}$ & $22 / 16 / 15$ & $\begin{array}{l}13.78 \pm 0.02 \\
(13.82)\end{array}$ & $\begin{array}{l}13.71 \pm 0.02 \\
(13.74)\end{array}$ & $\begin{array}{l}14.20 \pm 0.03 \\
(14.24)\end{array}$ & $15.46 \pm 0.03$ \\
\hline $\begin{array}{l}20180731 \\
5: 40-7: 32\end{array}$ & 33.61 & 32.65 & 0.542 & $\begin{array}{l}059 \\
54.5\end{array}$ & $10 / 10 / 9$ & $\begin{array}{l}13.98 \pm 0.030 \\
(13.96)\end{array}$ & $\begin{array}{l}14.02 \pm 0.025 \\
(14.02)\end{array}$ & $\begin{array}{l}14.38 \pm 0.029 \\
(14.38)\end{array}$ & $15.71 \pm 0.04$ \\
\hline 20190713 & 33.84 & 32.82 & 0.046 & 287 & $20 / 22 / 27$ & $13.96 \pm 0.024$ & $13.81 \pm 0.022$ & $14.23 \pm 0.025$ & $15.38 \pm 0.03$ \\
\hline
\end{tabular}

This article is protected by copyright. All rights reserved. 


\begin{tabular}{|l|l|l|l|l|l|l|l|l|l|}
\hline $7: 02-9: 22$ & & & & 55.9 & & $(13.96)$ & $(13.85)$ & $(14.28)$ & \\
\hline 20190714 & 33.84 & 32.82 & 0.020 & $231^{4}$ & $17 / 13 / 18$ & $\begin{array}{l}13.80 \pm 0.05 \\
(13.84)\end{array}$ & $\begin{array}{l}13.70 \pm 0.07 \\
(13.73)\end{array}$ & $\begin{array}{l}14.35 \pm 0.09 \\
(14.37)\end{array}$ & $15.43 \pm 0.05$ \\
$7: 05-9: 20$ & & & & 55.8 & & & \\
\hline
\end{tabular}

${ }^{1}$ Tidally locked: Charon sub-observer longitude $=$ Pluto sub-observer longitude $+180^{\circ}$. Longitudes are eastern longitudes.

${ }^{2}$ Reduced to mean opposition distance ( $r=39.482 \mathrm{AU}, \Delta=38.482 \mathrm{AU}$ ). No solar phase angle correction.

${ }^{3}$ Numbers in parenthesis are rotation-corrected using the lightcurve of Kosiarek (2016), as explained in the text.

${ }^{4}$ Pluto's "Heart-shaped" feature (Tombaugh Regio) visible.

Data reduction began using the IRAF analysis environment (Tody et al. 1993). Daytime dark frames of appropriate exposure times were coadded and subtracted from the domeflats and science images, which removed the majority of hot pixels. Flatfields at the JHK wavelengths were constructed, normalized, and ratioed with the science frames. Figure 1 shows a typical J-band Pluto/Charon frame after flat-fielding. With a plate-scale of 0.04 arcsec/pixel and with seeing of approximately 0.5 arcsec after the AO correction, the point-spread functions of Pluto and Charon were still convolved. We employed "ring masking" to separate Pluto and Charon fluxes. With ring-masking, a total of four images were generated from each science frame: a radially symmetric and a radially anti-symmetric image with Pluto as the centroid, and a radially symmetric and a radially anti-symmetric image with Charon as the centroid. To generate the radially symmetric image of Pluto for a given frame, the distance between each pixel and Pluto's centroid was measured and this radius was used to identify a list of pixels that fell along this radius. The median value of this list defined the radially symmetric component at this pixel. The radially antisymmetric image is defined as the original image minus the radially symmetric image. Aperture photometry for these four images was used to measure the Pluto and Charon fluxes with a photometric aperture of 30 pixels in radius and a sky annulus with an outer radius of 60 pixels and an inner radius of 30 pixels.

Note that a point-spread function fitting technique was not used for two reasons: the brightness of the standard star and Pluto was greatly different on most nights; and with adaptive optics, the PSF changes with position on the image and throughout the night.

To convert our instrumental magnitudes into calibrated magnitudes we performed aperture photometry of the brightest background field stars in our frames, which enabled a calibration even under non-photometric conditions. The JHK magnitudes from background reference stars were found using the VizieR Catalog (VizieR catalogue access tool: https://vizier.u-strasbg.fr/vizbin/VizieR) and are from the 2MASS Survey (Skrutskie et al. 2006; see Table 2). The error budget for the calibrated magnitudes for Pluto and Charon listed in Table 1 included the dispersion in the individual frames (typically 10+ measurements per filter per night), and the errors listed in the 2MASS cataloged magnitudes, each added in quadrature. Finally, the resulting magnitudes in each filter for both objects were averaged on a given night; the number of images averaged for each filter is listed in Table 1. The excursion in solar phase angle was not sufficient on any night to show a trend in brightness; the largest span was on July 12, 2018, from 0.011 to 0.0084 . The signal for Charon was sufficient only in the J-filter to construct a solar phase curve. The listed photometric uncertainties of the cataloged on-chip reference stars dominated our error budget; the errors are especially large for the dimmer stars. In no case was there more than one photometric standard star in our field.

This article is protected by copyright. All rights reserved. 
Table 2 - Background reference stars

$\begin{array}{ccccc}\text { UT Date } & \text { Catalog ID } & \text { J } & \text { H } & \text { K } \\ 20180712 & \text { XPM 136-1294179 } & 15.232 \pm 0.046 & 14.943 \pm 0.090 & 14.858 \pm 0.109 \\ 20180713 & \text { XPM 136-1293908 } & 12.922 \pm 0.023 & 12.334 \pm 0.022 & 12.289 \pm 0.027 \\ 20180731 & \text { XPM 136-1290301 } & 12.686 \pm 0.030 & 12.106 \pm 0.025 & 12.027 \pm 0.027 \\ 20190713 & \text { XPM 135-1364022 } & 12.593 \pm 0.023 & 12.248 \pm 0.022 & 12.189 \pm 0.024 \\ 20190714 & \text { XPM 135-1363813 } & 15.448 \pm 0.052 & 14.878 \pm 0.068 & 14.782 \pm 0.091\end{array}$
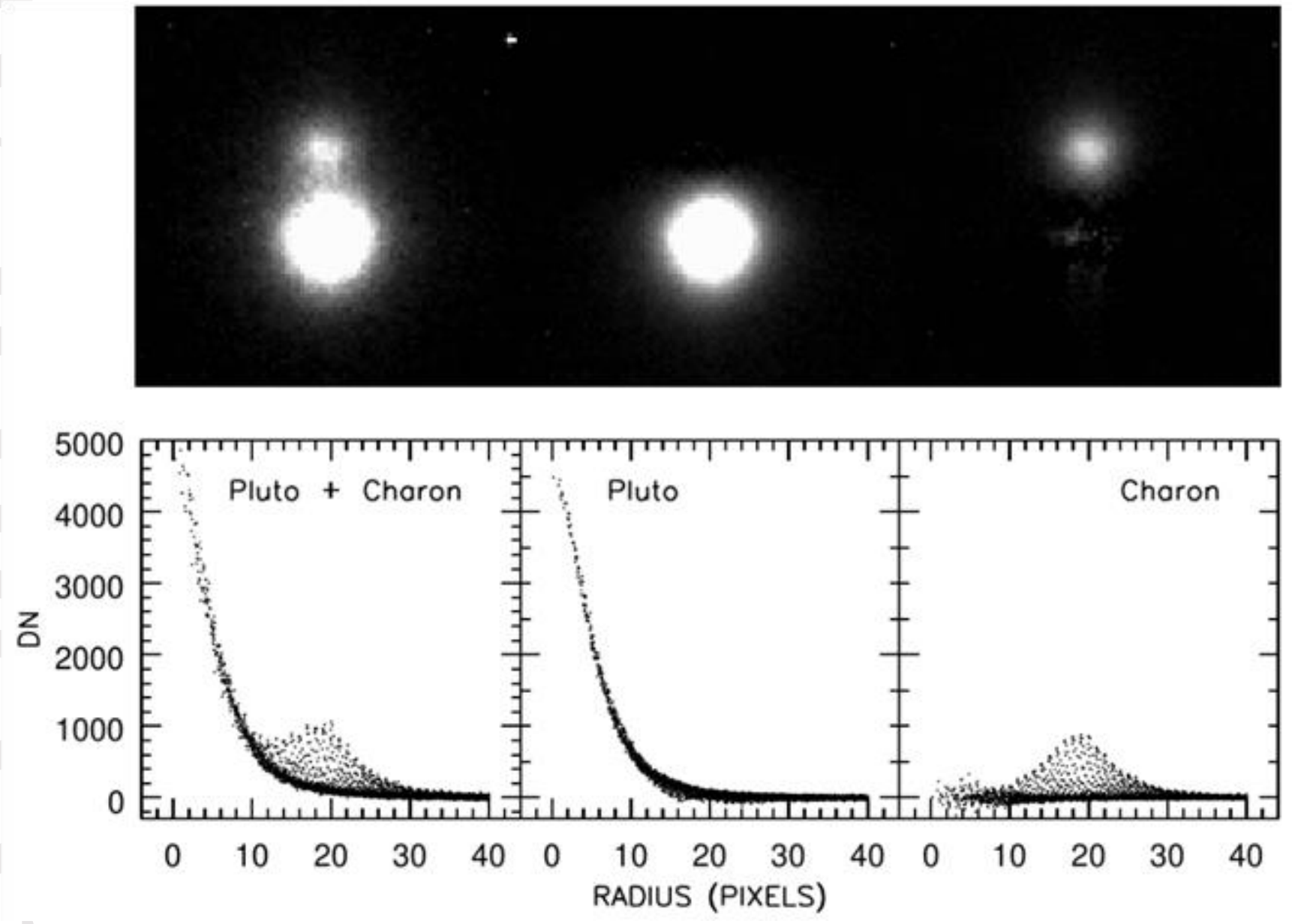

Figure 1. Efficacy of ring-masking in the deconvolution of the Pluto and Charon point-spread functions. The upper panel shows a typical J-band image, obtained 201807 12 7:30 UT. The next two components in the upper panel show the radially anti-symmetric image centroided around Charon (which isolates the flux of Pluto) and the radially anti-symmetric image centroided around 
Pluto (which isolates the flux of Charon). The bottom panel shows the radial profiles centered about Pluto, for comparison.

We also made corrections to our observations of Pluto to account for changes in brightness due to rotational solar phase variations. Pluto, and to a lesser extent Charon, exhibit changes in brightness as a function of subobserver longitude (e.g., Buie et al., 2010; Buratti et al., 2015; 2019). Our observations are not extensive enough to determine a rotational phase curve. However, we were able to make use of the JHK rotational lightcurve of Kosiarek (2016) to correct for this effect, which is not large; the rotation-corrected magnitude for each data point is listed in parentheses under each magnitude listed in Table 1 and shown in Figure 2 by the red data points. These corrections are from Figure 3-5 in Kosiarek (2016), which gives JHK model lightcurves for the years 2015 to 2065 in the case of a static frost model for Pluto. We used the same numbers for 2018-2019, as these amplitudes change very slowly. For Charon, which does not have a published light curve in the near-IR, the rotational effect should be even smaller, as its lightcurve in the New Horizons MVIC NIR -filter has a half-amplitude of about 0.03 magnitudes (Buratti et al., 2019). The center of this filter's bandpass is at $\sim 0.94 \mu \mathrm{m}$, so corrections to our longer-wavelength groundbased measurements cannot be made directly from data collected with it. However, the visible rotational lightcurve is due mostly to differences in water ice abundance on the surface (Howett et al. 2017; Buratti et al., 2019). The spectral contrast between opaque material and water ice decreases at longer wavelengths: the rotational corrections for Charon would thus be at most $\sim 3 \%$.

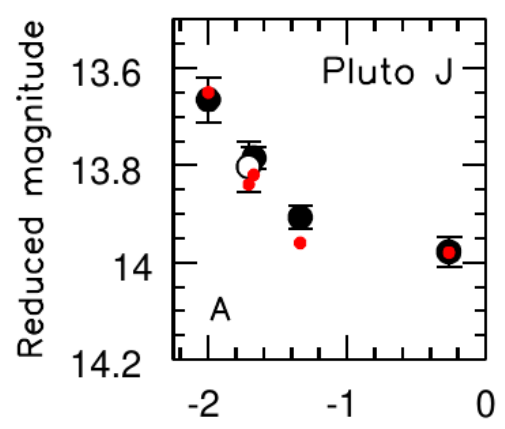

$\log _{10}$ Solar phase angle

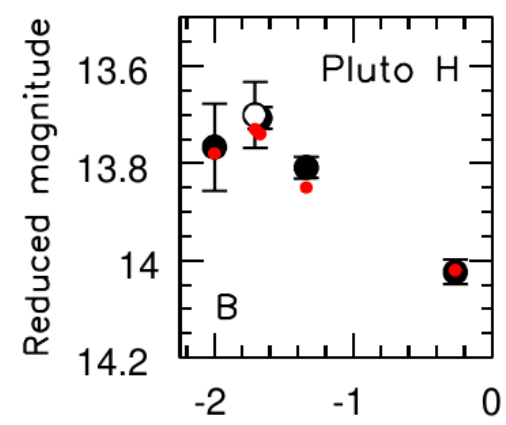

$\left({ }^{\circ}\right)$

$\log _{10}$ Solar phase angle
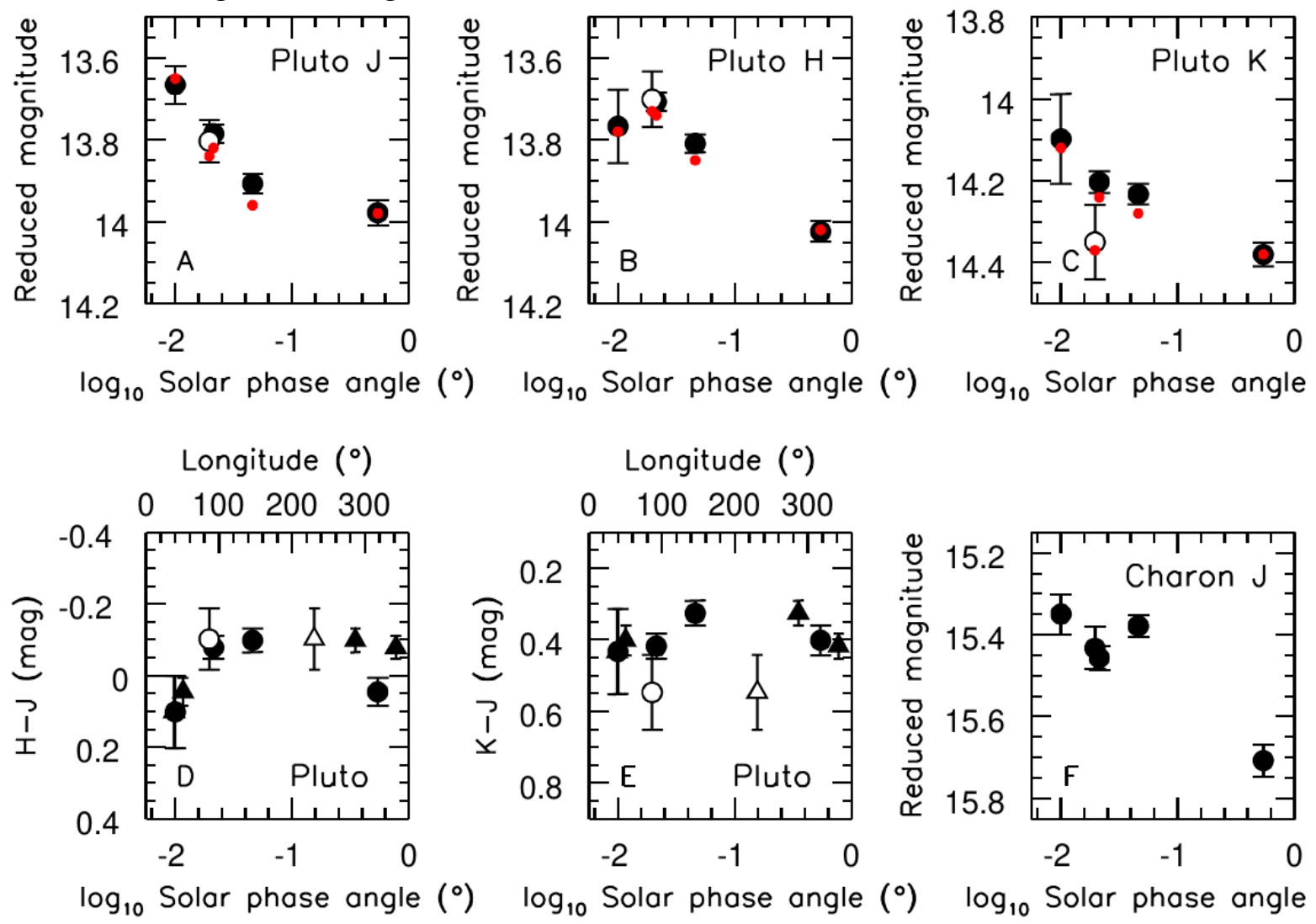

$\left({ }^{\circ}\right)$

$\log _{10}$ Solar phase angle $\left({ }^{\circ}\right)$
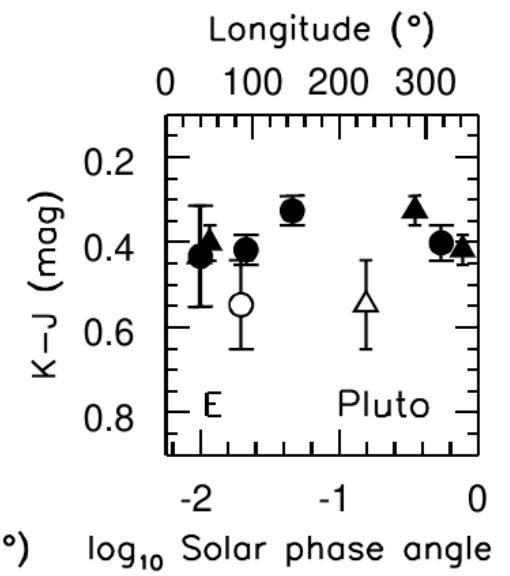

$\left({ }^{\circ}\right)$

$\log _{10}$ Solar phase angle

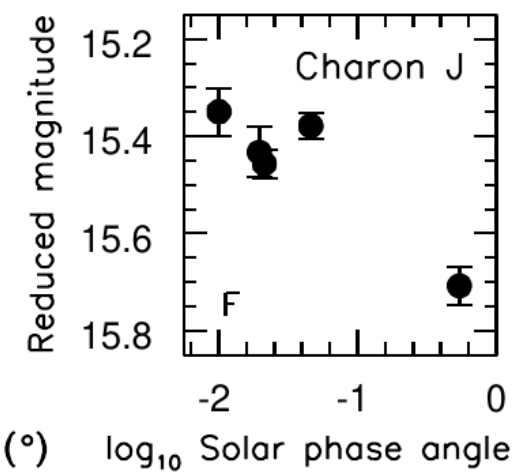

$\left({ }^{\circ}\right)$

Figure 2. The reduced magnitudes as a function of solar phase angle, with red points representing data that have been corrected for rotational phase variations using the light curve of Kosiarek (2016). Figures A, B, and C show the phase curves for Pluto; D and E show colors; and F shows the J-filter data for Charon. In the top three cells, the open point shows data that were gathered

This article is protected by copyright. All rights reserved. 
when Tombaugh Regio was visible. For the graphs showing H-J and K-J colors (D and E), the longitude (with the scale at the top) is shown in diamonds while the solar phase angle (with the scale at the bottom), is shown in circles with Tombaugh Regio again with open symbols. Figures $2 \mathrm{D}$ and $2 \mathrm{E}$ show there is no discernable color dependence to the opposition surge. Although the data are noisy, Charon also appears to exhibit a huge surge (Figure 2F).

Because Pluto's hazy atmosphere is so extraordinarily forward-scattering, its photometric effects are not apparent until much larger phase angles (Hillier et al., 2021).

\section{Results}

Figure 2 shows the results for all three filters for Pluto, and for Charon in the J-filter, and colors for Pluto. In the J-filter, Pluto's solar phase curve increases by 0.31 magnitudes, a $33 \%$ increase in brightness. Observations in the H-filter and K-filter show similar increases of 0.32 mags and 0.28 mags, respectively, although the data are more noisy. Charon's surge is slightly higher $(\sim 0.36$ magnitudes), with a possibly broader shape. Further observations assigned or requested between June and October 2021 will clarify (if successful) the shape of both surges and extend the curve to larger solar phase angles (the maximum attainable currently is $1.67^{\circ}$ ).

As stated above, our errors are dominated by the errors in the photometric accuracy of the standard stars. These errors are reflected in the data points at the two lowest solar phase angles in the $\mathrm{H}$ and $\mathrm{K}$ filters, where errors of $\sim 0.10$ are stated. Unfortunately, these relatively dim standard stars were in the field on these two prime nights, but fortunately, the stars' measurements in the J-filter had smaller errors with a more solid result.

\section{Summary, discussion and future work}

We successfully captured a unique opportunity to observe the Pluto-Charon system at its smallest solar phase angle visible from Palomar Observatory, which will not be repeated for another 161 years. Data from the PHARO adaptive optics system on the 200 -inch in the J-filter show that Pluto exhibits a surge of over 0.3 magnitudes in the JHK filter system. Our observations so far show no clear color dependence on solar phase angle or longitude. Notably, Tombaugh Regio - the "heart" of Pluto - exhibits the same surge-amplitude in $\mathrm{J}$ and $\mathrm{H}$, and within the error bars for $\mathrm{K}$, as the opposite, lower albedo hemisphere. This result suggests that the surface processes causing this surge, which possibly include ongoing deposits of volatiles and haze particles from the atmosphere, are global. Although data for Charon are noisier, it exhibits a 0.36 magnitude increase in brightness in its last half-degree of solar phase angle.

Because our observations of the Pluto system's solar phase curve are among the first in the JHK spectral range, it is difficult to make comparisons with other objects. When compared with visible measurements, our data show that Pluto and perhaps Charon exhibit surges that are among the largest in the Solar System. Rabinowitz et al. (2007) observed 10 KBOs and Centaurs below a solar phase angle of 0.10 , and none showed such a large surge. Varuna increases 0.16 magnitudes between $0.5^{\circ}$ and $0.01^{\circ}$ (Hicks et al., 2005). Triton's surge over the same range is $\sim 0.2$ magnitudes at $0.89 \mu \mathrm{m}$ (Buratti et al., 2011). Perhaps the most similar extant data are those that show the Uranian moon Ariel with a visible opposition surge of $\sim 0.3$ magnitudes between $0.5^{\circ}$ and opposition (Karkoschka, 2001). The most comparable published data set of an outer planet moon or small body was obtained by the Cassini Visual Infrared Mapping Spectrometer (VIMS), with a wavelength range of 0.3-5.1 $\mu \mathrm{m}$ (Brown et al., 2004). Extensive observations of the moons of

This article is protected by copyright. All rights reserved. 
Saturn during the orbital tour showed increases of about 0.20 magnitudes in the last 0.5 degree, although the minimum angle was only 0.05 (Buratti et al., 2009). Figure 3 compares observations from Rhea with those of Pluto in the J-filter: not only is the latter's surge much larger, it is sharper.

One factor that limits the minimum solar phase angle attainable is the angular size of the Sun at the distance of the target. It is possible that the geometric effect of a smaller solar phase angle achievable because of the smaller angular size of the Sun may contribute to a larger brightness surge. But opposition solar phase curves of several Kuiper Belt Objects were captured at solar phase angles less than $0.10^{\circ}$, and Nereid, the moon of Neptune was observed as low as $0.01^{\circ}$, and their solar phase curves are more in line with those of most of the icy moons and small bodies, with 0.20 mag increase in the last half-degree; they do not show as strong a surge as that observed on Pluto/Charon. Furthermore, Pluto's surge, and possibly Charon's, is unusually sharply peaked. Thus there appears to be something unusual about Pluto. Perhaps its optically active regolith is comprised of smaller grain sizes due to active seasonal volatile transport, ongoing geological processes, or even snow. Charon, which currently has no atmosphere or ongoing geologic activity, may accrete particles from Pluto's atmosphere to provide a uniquely textured regolith. Such an accumulation - with subsequent photolysis - was hypothesized to explain its reddish polar cap (Grundy et al., 2016b). Verbiscer et al. (2007) observed large surges in the visible for the Saturnian moons. They attributed this effect to micron-sized particles from Saturn's E-ring, which suggests a similar phenomenon could be occurring on their surfaces.

Estimates of the sizes of Pluto's surface particles vary widely. Protopapa et al. (2017) estimate sizes in the range of 10-1000 $\mu \mathrm{m}$. Hillier et al. (2021) model atmospheric aerosols to be micronsized, as do Gladstone et al. (2016). The surface of Pluto appears to be covered with particles from its atmosphere (Stern et al., 2015; Moore et al. 2016), and it is reasonable to assume that these particles are small based on the atmospheric models.

Although additional observations between solar phase angles of $0.54^{\circ}$ (our maximum solar phase angle) and $1.67^{\circ}$ (the maximum solar phase angle observable from Earth in the next two years) are required to better model the opposition surge, our initial results appear to be a validation of Hapke's coherent backscatter model. A radiative transfer model was fit to a solar phase curve compiled from ground-based and New Horizons data (Hillier et al., 2021). The model predicts a very large surge near opposition comparable to the one observed, based on the importance of coherent backscatter. This correlation is only notional, as the Hillier et. al model was fit to visible data. (The additional observations required for modeling were planned for 2020, but Palomar Observatory shut down for COVID-19 during Pluto's entire 2020 apparition; it is not observable again until June 2021. Additional observations are assigned in summer 2021 and observations at large solar phase angles are being requested in August and September 2021.) These are the first near-IR solar phase curves for the Pluto-Charon system, but an instrument on New Horizons, LEISA, gathered near-infrared data at mid- and high- solar phase angles. Although the purpose of these data were to understand the composition of the system's bodies, a solar phase curve could be produced from the data to augment the observations presented here.

This article is protected by copyright. All rights reserved. 


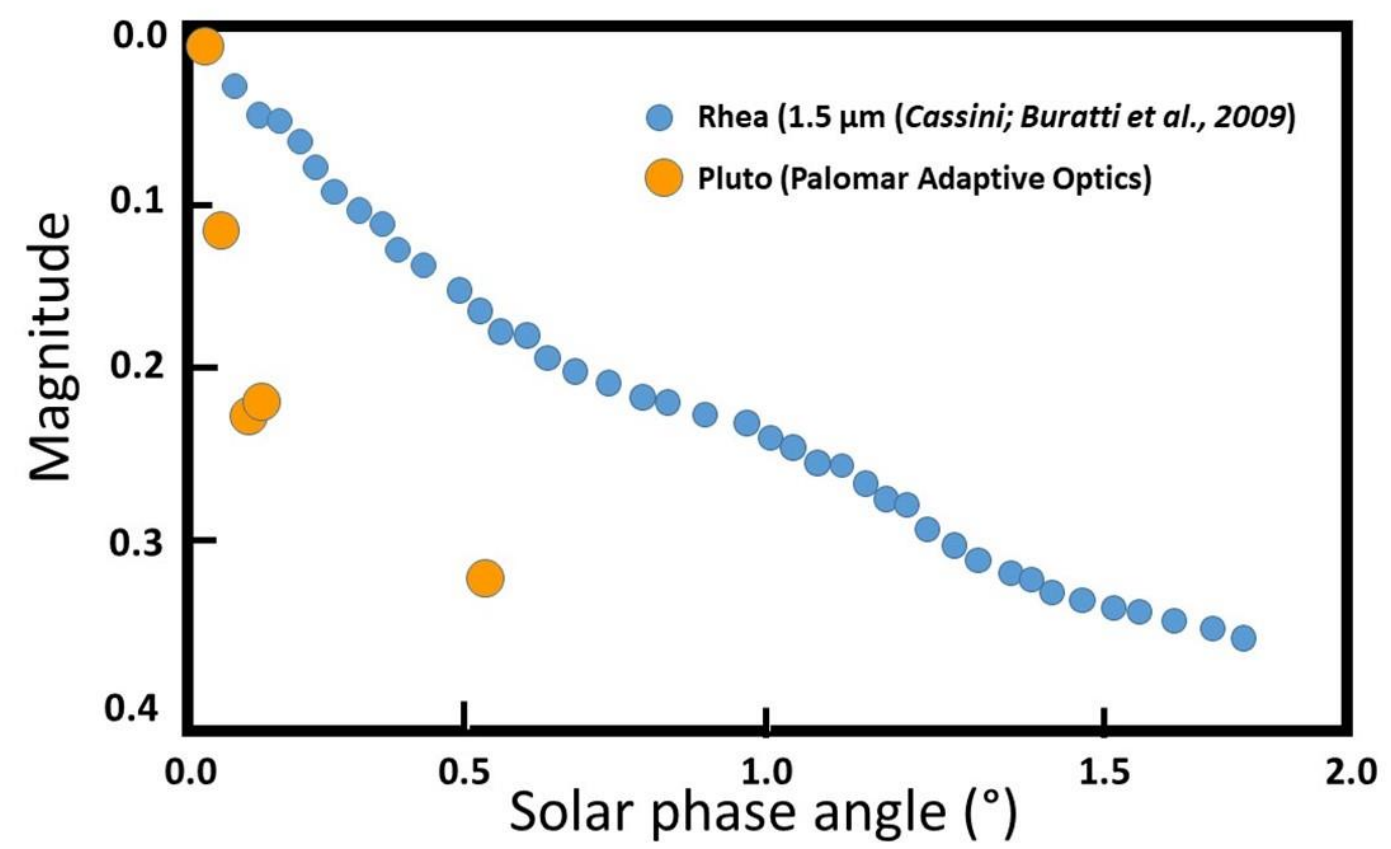

Figure 3. Our Pluto J-filter data compared with a phase curve of Rhea from the Cassini VIMS instrument at the same wavelength (Buratti et al., 2009). Not only is Pluto's surge among the largest in the Solar System, it appears to have a sharper peak, similar to that modeled by Hillier et al (2021) with visible measurements.

Observations at very small phase angles enable an accurate calculation of the geometric albedo, which is the integral brightness of a planetary body at opposition compared to a diffuse, Lambert disk of the same projected area. This quantity is important for understanding energy transport on a planetary body: when combined with the phase integral it yields the Bond albedo, the fundamental measure of energy balance, which is an important parameter to measure for an object that exhibits seasonal volatile transport and active geology. Making use of Equation (2) in Buratti et al. (2015) with the rotation corrections of Kosiarek (2016) yields geometric albedos of $0.86 \pm 0.04,0.59 \pm 0.05$, and $0.39 \pm 0.04$ in the JHK filters, respectively. Charon's geometric albedo in the J-filter is $0.68 \pm 0.06$, without rotation corrections (half our error is due to poor knowledge of Charon's rotation curve). For comparison, the visible albedo of Pluto is $0.59 \pm 0.04$ (Buratti et al., 2015), or as high as $\sim 1.0 \pm 0.1$ if the opposition surge modeled by Hillier et al. (2021) is included. Charon's albedo at $0.61 \mu \mathrm{m}$ is $0.41 \pm 0.01$ (Buratti et al., 2017).

\section{References}

Brown, R. H., \& Cruikshank, D. P. (1983). The Uranian satellites: Surface compositions and opposition brightness surges. Icarus 55, 83-92.

Brown, R. H, Baines, K. H., Bellucci, G., Bibring, J.-P., Buratti, B. J., Capaccioni, F. et al. (2004). The Cassini visual and infrared mapping spectrometer (VIMS) investigation. Space Science Reviews 115, 111-168.

Buie, M. W., Grundy, W M., Young, E. F., Young, L. A., \& Stern, S. A. (2010). Pluto and Caron

This article is protected by copyright. All rights reserved. 
with the Hubble Space Telecsope. II. Monitoring Global Change and Improved Surface Properties from Light Curves. Astronomical Journal 139, 1117-1127.

Buratti, B. J., Gibson, J. \& Mosher, J. (1992). CCD observations of the Uranian satellites. Astronomical Journal 104, 1618-1622.

Buratti, B. Hillier, J., \& Wang, M. (1996). The lunar opposition surge: observations by Clementine, Icarus $124,490-499$.

Buratti, B. J., Mosher, J. A., Abramson, L., Akhter, N., Clark, R. N., Brown, R. H. et al. 2009. Opposition surges of the satellites of Saturn from the Cassini VIMS. LPSC \#40 ID\#1738.

Buratti, B. J., Bauer, J. M., Hicks, M. D., Hillier, J. K., Verbiscer, A., Hammel, H., et al. (2011). Photometry of Triton 1992-2004: Surface volatile transport and discovery of a remarkable opposition surge. Icarus 212, 835-846.

Buratti, B. J., Hicks, M. D., Dalba, P. A., Chu, D., O’Neill, A., Hillier, J. K. et al. (2015). Photometry of Pluto 2008-2014: Evidence of ongoing seasonal volatile transport and activity. Astrophysical Journal Letters 804:L6.

Buratti, B. J., Hofgartner, J. D., Hicks, M. D., Weaver, H. A., Stern, S. A., Momary, T. et al. (2017). Global albedos of Pluto and Charon from LORRI New Horizons observations. Icarus 287, 207-217.

Buratti, B. J., Hicks, M. D., Hillier, J. H, Verbiscer, A. J., Abgarian, M., Hofgartner, J. D., et al. (2019). New Horizons Photometry of Pluto's Moon Charon. Astrophysical Journal Letters 874, article id. L3, DOI: 10.3847/2041-8213/ab0bff

Dekany, R., Roberts, J., Burress, R., Bouchez, A., Truong, T., Baranec, C., et al. (2013). PALM3000: Exoplanet Adaptive Optics for the $5 \mathrm{~m}$ Hale Telescope. The Astrophysical Journal 776. doi:10.1088/0004-637X/776/2/130

Domingue, D. 1., Hapke, B., Lockwood, G. W., \& Thompson, D. T. (1991). Europa's phase curve: Implications for surface structure. Icarus 90, 30-42.

Gladstone, G. R., Stern, S. A, Ennico, K., Olkin, C. B., Weaver, H. A., Young, L. A., et al. (2016). The Atmosphere of Pluto as observed by New Horizons. Science 351, aad8866.

Grundy, W.M., Binzel, R. P., Buratti, B. J., Cook, C. C., Cruikshank, D. P., Dalle Ore, C. M. et al. (2016a). Surface compositions across Pluto and Charon. Science 351, id.aad9189.

Grundy, W.M., Cruikshank, D. P., Gladstone, G. R., Howett, C. J. A., Lauer, T. R., Spencer, J. R. et al. (2016b). The formation of Charon's red poles from seasonally cold-trapped volatiles. Nature $539,65-68$.

Hapke, B. (1986). Bidirectional reflectance spectroscopy. 4. The extinction coefficient and the opposition effect. Icarus 67, 264-280.

This article is protected by copyright. All rights reserved. 
Hapke, B. (2008). Bidirectional reflectance spectroscopy. 6. Effects of porosity. Icarus 195, 918926.

Hapke, B. (1990). Coherent backscatter and the radar characteristics of outer planet satellites. Icarus 88, 407-417.

Hapke, B. (2009). A quantitative test of the ability of models based on the equation of radiative transfer to predict the bidirectional reflectance of a well-characterized medium. Icarus 199, 210218.

Hayward, T. L., Brandl, B., Pirger, B., Blacken, C., Gull, G. E., Schoenwald, J., \& Houck, J. R. (2001). PHARO: A Near-Infrared Camera for the Palomar Adaptive Optics System. Publications of the Astronomical Society of the Pacific 113, 105-118.

Hicks, M., D. Simonelli, \& B. Buratti (2005). Photometric behavior of 20000 Varuna at very small solar phase angles. Icarus 176, 492-498.

Hillier J. K., Buratti, B. J., Hofgartner, J. D., Hicks, M. D., Devins, S. \& Kivrak, L. (2021). Characteristics of Pluto's haze and surface from an analytic radiative transfer model. Planetary Sciences Journal 2, 11 https://doi.org/10.3847/PSJ/abbdaf

Howett, C. J. A., Ennico, K., Olkin, C. B., Buie, M. W., Verbiscer, A. J., Zangari, A. M. et al. (2017). Charon's light curves, as observed by New Horizons' Ralph color camera (MVIC) on approach to the Pluto system Icarus 287, 152. doi: 10.1016/j.icarus.2016.09.031

Irvine, W. M. (1966). The shadowing effect in diffuse radiation. Journal of. Geophysical. Research 71, 2931-2937.

Karkoschka, E. (2001). Comprehensive Photometry of the Rings and 16 Satellites of Uranus with the Hubble Space Telescope. Icarus 151, 51-68. doi: 10.1006/icar.2001.6596

Kosiarek, M. (2016). Modeling Pluto's Light Curve in the Near Infrared: Implications for Observation Post New Horizons. M. S. Thesis, Massachusetts Institute of Technology, Cambridge, MA

Lisse, C., McNutt, R. L., Jr., Wolk, S. J., Bagenal, F., Stern, S. A., Gladstone, G. R.et al. (2017). The puzzling detection of x-rays from Pluto by Chandra. Icarus 287, 103-109.

Mishchenko, M. I. (1992). The angular width of the coherent backscatter opposition effect: An application to icy outer planet satellites. Astrophysics and Space Science 194, 327-333.

Mishchenko, M. I. Dlugach, J. M., Liu, L., Rosenbush, V. K., Kiselev, N. N., Shkuratov, Y. G. (2009). Direct Solutions of the Maxwell Equations Explain Opposition Phenomena Observed for High-Albedo Solar System Objects. Astrophysical Journal Letters 705, L118-122.

Moore, J. M., McKinnon, W. B., Spencer, J. R., Howard, A. D., Schenk, P. M., Beyer, R. et al. (2016). The geology of Pluto and Charon through the eyes of New Horizons. Science 351, 12841293.

This article is protected by copyright. All rights reserved. 
Ozrin, V.D. (1992). Exact solution for coherent backscattering of polarized light from a random medium of Rayleigh scatterers. Waves in Random Media 2, 141-164.

Protopapa, S., Grundy, W. M., Reuter, D., Hamilton, D. P., Dalle Ore, C. M., Cook, J. C. et al. (2017). Pluto's global surface composition through pixel-by-pixel Hapke modeling of New Horizons Ralph/LEISA data. Icarus 287, 218-228. doi: 10.1016/j.icarus.2016.11.028

Rabinowitz, D. L., Schaeffer, B., \& Tourtellotte, S. W. (2007). The Diverse Solar Phase Curves of Distant Icy Bodies. I. Photometric Observations of 18 Trans-Neptunian Objects, 7 Centaurs, and Nereid. Astronomical Journal 133, 26-43.

Skrutskie, M. F., Cutri, R. M., Stiening, R., Weinberg, M. D., Schneider, S., Carpenter, J. M. et al. (2006). The Two Micron All Sky Survey (2MASS). The Astronomical Journal 131, 1163-1183. doi:10.1086/498708

Stern, S. A., Bagenal, F., Ennico, K., Gladstone, G. R., Grundy, W. M., McKinnon, W. B. et al. (2015). The Pluto System: Initial Results from its Exploration by New Horizons. Science 350, 292.

Tody, D., 1993. IRAF in the Nineties, in Astronomical Data Analysis Software and Systems II, A.S.P. Conference Ser., Vol 52, eds. R.J. Hanisch, R.J.V. Brissenden, \& J. Barnes, 173.

Verbiscer, A., French, R., Showalter, M., \& Helfensein, P. (2007). Enceladus: Cosmic Graffiti Artist Caught in the Act. Science 315, 815.

https://www.astro.caltech.edu/palomar/observer/200inchResources/pharocookbook.html

\section{Acknowledgements}

This research was funded by the NASA Solar System Observations Program and the JPL Fellows Program, and carried out at the Jet Propulsion Laboratory, California Institute of Technology, under contract to the National Aeronautics and Space Administration. We appreciate the support of the Palomar Time Allocation Committee, especially Michael Werner and Robert Brucato, who agreeably accommodated our difficult scheduling requests, and to the Palomar Observatory staff, especially Rick Burress, Carolyn Heffner, and Kajsa Peffer.

The astronomical images obtained for this study are in the process of being archived in the Planetary Data System Small Bodies Node at https://doi.org/10.26007/feev-qn61.

This article is protected by copyright. All rights reserved. 


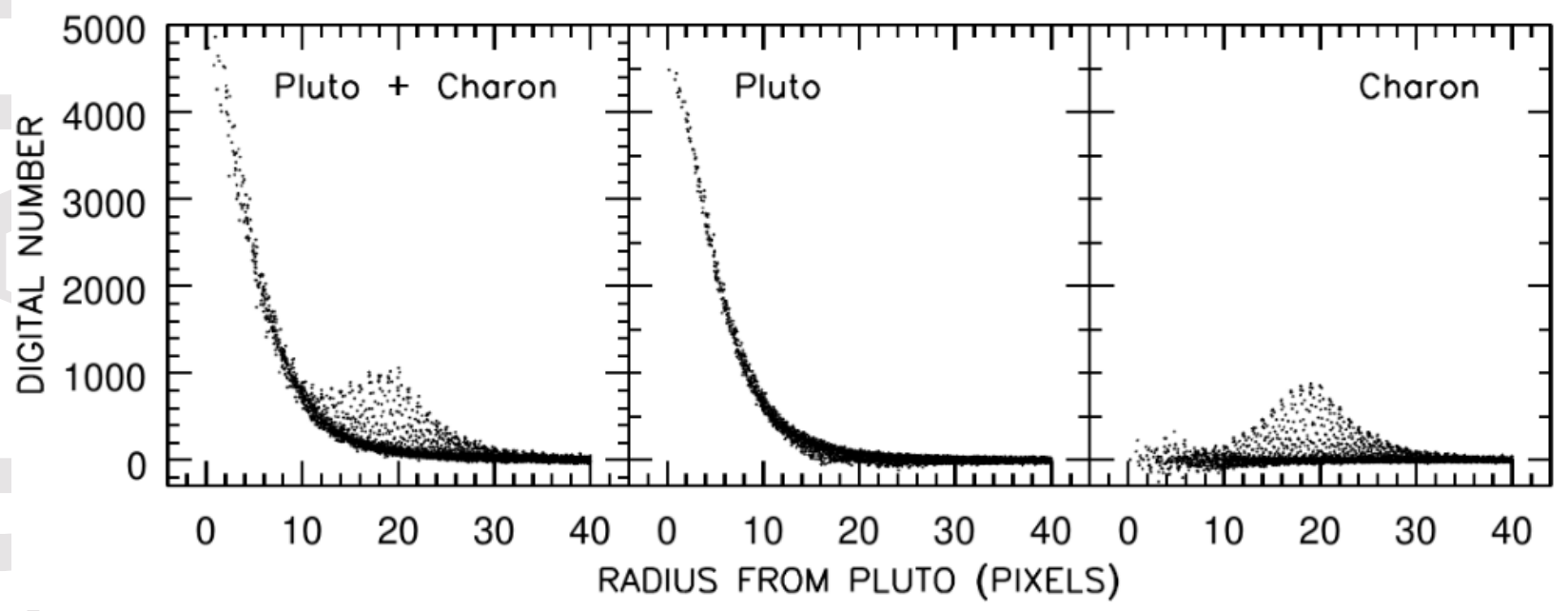

This article is protected by copyright. All rights reserved. 

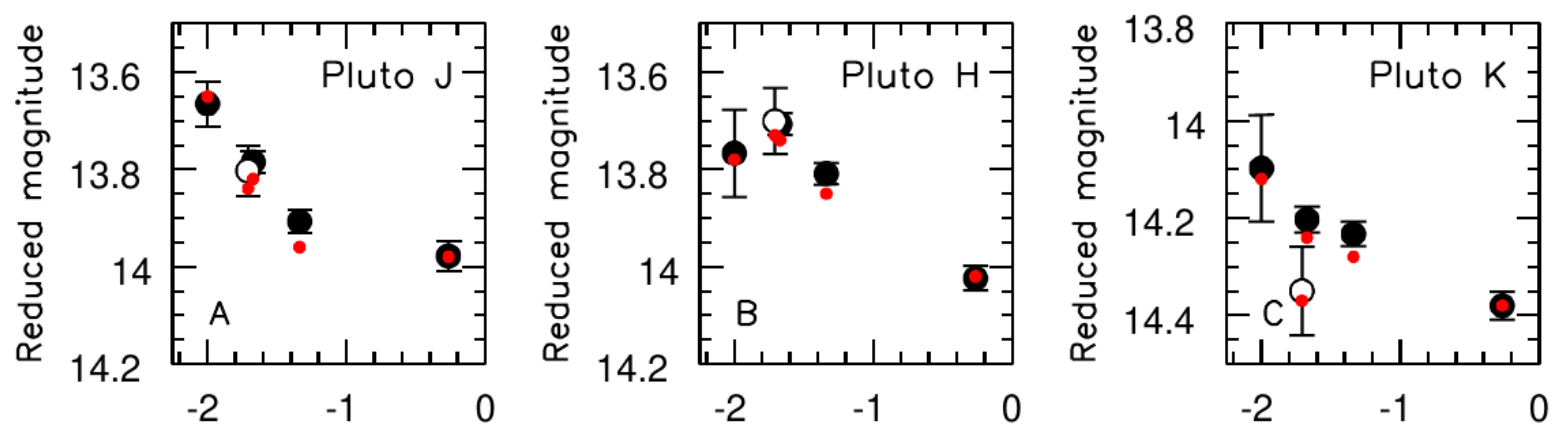

$\log _{10}$ Solar phase angle

$\left({ }^{\circ}\right) \quad \log _{10}$ Solar phase angle

$\left({ }^{\circ}\right) \quad \log _{10}$ Solar phase angle $\left({ }^{\circ}\right)$
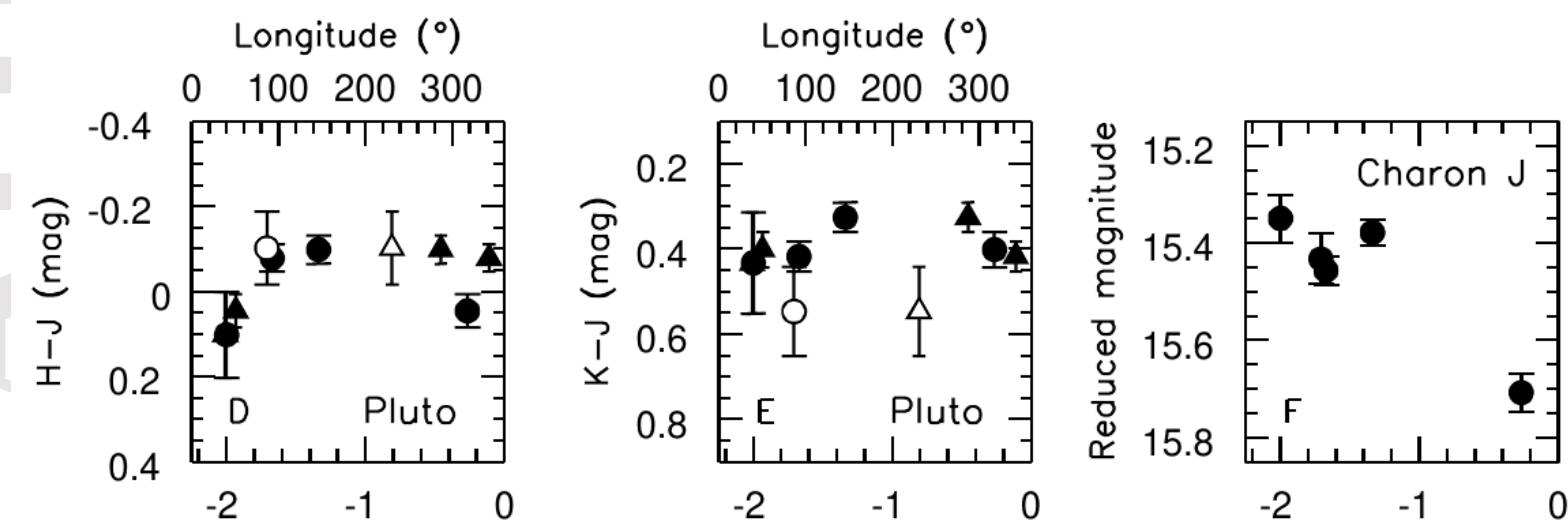

$\log _{10}$ Solar phase angle $\left(^{\circ}\right) \quad \log _{10}$ Solar phase angle $\left(^{\circ}\right) \log _{10}$ Solar phase angle $\left(^{\circ}\right)$

This article is protected by copyright. All rights reserved. 


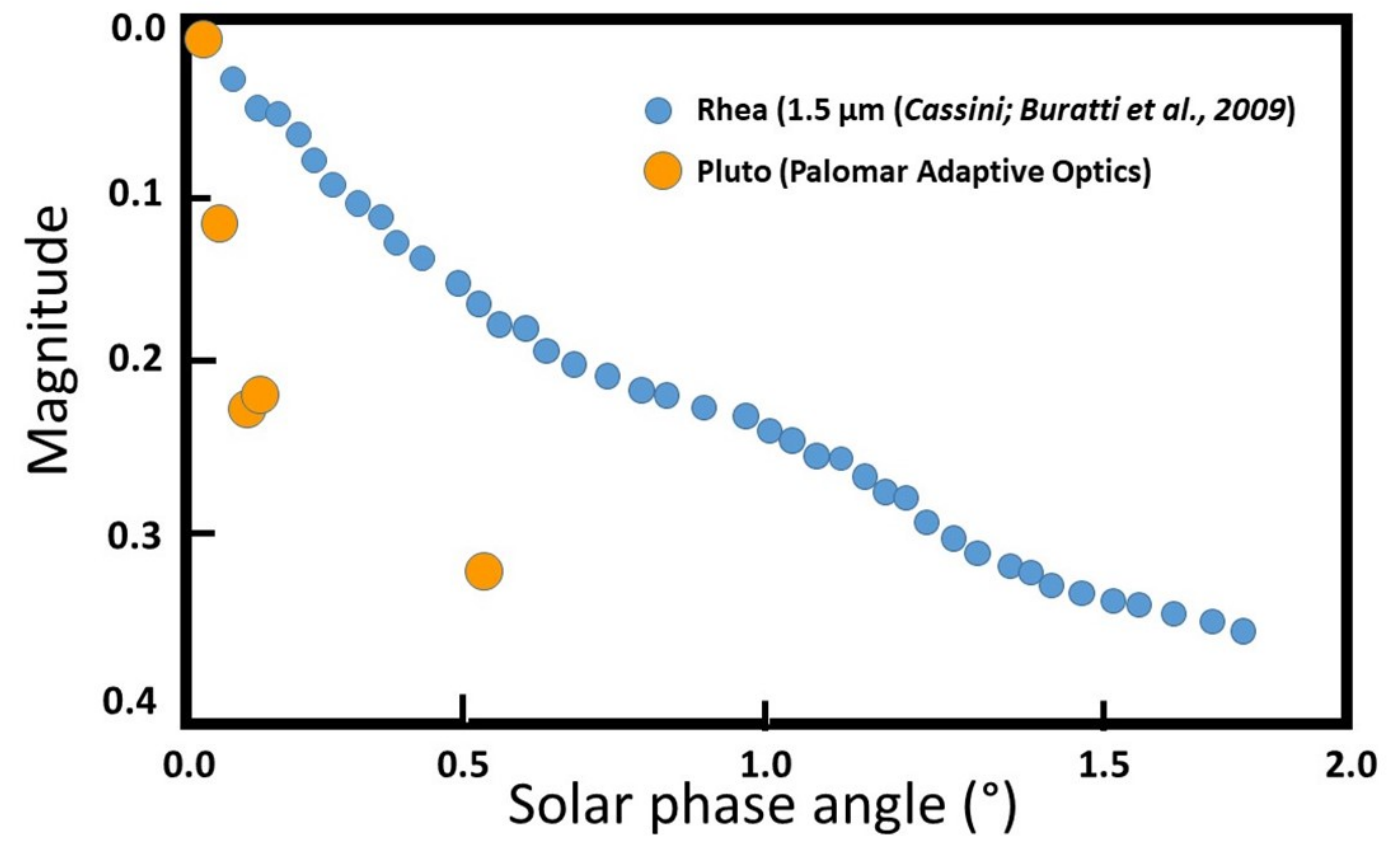

This article is protected by copyright. All rights reserved. 